
\title{
25 Research Square \\ FOXO3a shRNA and simultaneous induction of P27Kip1gene Inhibit the Breast Cancer Growth in Nude Mice
}

\section{Sabah Mayahi}

Golestan University of Medical Sciences and Health Services

Masood Golalipour

Golestan University of Medical Sciences and Health Services

Mohammad Ashari

Golestan University of Medical Sciences and Health Services

Majid Shahbazi ( $\square$ shahbazim@goums.ac.ir)

Golestan University of Medical Sciences and Health Services https://orcid.org/0000-0002-4143-8282

Research article

Keywords: F0X03a gene, P27Kip1 gene, Breast cancer, Gene therapy

Posted Date: June 16th, 2020

DOI: https://doi.org/10.21203/rs.3.rs-31573/v1

License: (c) (i) This work is licensed under a Creative Commons Attribution 4.0 International License.

Read Full License 


\section{Abstract}

Background: FOXO proteins, which are overexpressed in multiple human tumors, belong to the Forkhead family of transcription factors that are involved in cell-cycle regulation, cell apoptosis, differentiation, stress response, and metabolism. The p27Kip1 gene leads to cell cycle arrest, cell apoptosis, tumor suppressor genes, and cell adhesion. The low expression level of the p27Kip $1 \mathrm{gene}$ is attributed to poor prognosis in patients with colorectal, gastric, pulmonary, and breast cancers. Accordingly, the present study aimed to investigate the possibility of tumor growth inhibition in a mouse model by targeting FOXO3a shRNA and the simultaneous induction of P27Kip 1gene.

Methods: The tumor model was generated by intratumoral inoculating with plasmids. When tumor size reached an average volume of $8 \mathrm{~mm}$ in diameter, the mice received injections of construct and control plasmids three times a week for two weeks, followed by tumor growth assessment.

Results: Based on the obtained results, the delivery of construct plasmid significantly inhibited tumor growth in nude mice, as compared to the control plasmid. Moreover, the immunohistochemical analysis indicated that the delivery of construct plasmid significantly suppressed expression of FOXo3a and induced P27Kip1 in tumor samples.

Conclusion: The findings of the present study revealed that FOXO3a shRNA, along with simultaneous induction of P27Kip1gene using a useful in vivo gene delivery strategy, seems a practical therapeutic approach for breast cancer treatment and may provide profound insight into gene therapy of solid cancers.

\section{Background}

Breast cancer is the most prevalent cancer in women worldwide, which contributes to nearly 1.7 million new cases diagnosed accounts for than 520,000 related deaths just in 2012(1). Triple-negative breast cancer (TNBC), which is highly aggressive, attributes to $15 \%$ of breast cancer incidence rates. This disease, which mostly affects younger patients, is characterized by tumors that lack expression of estrogen receptor(ER) progesterone receptor and HER2, as well as a poor clinical prognosis (2). Expression of some genes, such as P27Kip 1 and forkhead box 03 (FOXO3), changes in breast cancer. A low $P 27 k i p 1$ level can reduce the survival of cancer patients (3). The reduced $p 27 K i p 1$, which is a crucial regulator of G1-to-S phase progression, is closely connected with high histopathologic tumor grade (4). The forkhead box 03 (FOXO3a) transcription factors act as relevant regulators of cellular proliferation, cell cycle arrest, apoptosis, autophagy, and metabolism(2). As researchers indicated, FOXO3a inactivation may efficiently prevent tumor expansion and metastasis $(5,6)$.

Loss of sensitivity to chemotherapy and a poor prognosis resulted in cancer recurrence in many patients with advanced breast cancer (1). Metastasis develops breast cancer into an incurable disease with a median survival time of around two years. In this stage, chemotherapy is the mainstay of treatment. A response rate of $35 \%-67 \%$ has been reported for combined chemotherapy with a short median response 
duration of about nine months. Accordingly, it seems essential to find alternative therapies for patients who are inflicted with chemotherapy or hormone-refractory cancers. Gene therapy can be regarded as an alternative therapy in this regard (7). Since in vivo studies of human breast cancer development in 1969 and the nude mice has been increasingly used in cancer research (8). In the current study, we designed a bidirectional construct that associated FOXO3a-shRNA and overexpression P27Kip1 simultaneously; after that, we investigated whether the constructed plasmid can suppress tumor growth in a nude mouse.

\section{Methods}

\section{Constructed plasmid}

A short hairpin RNA (shRNA) targeting FOXO3a along with simultaneous induction of P27Kip1 gene (Figure 1) were designed, synthesized (BioMatic, Canada), and inserted into the blank expression plasmid (PcDNA3. $1^{+}$expression vector with EGFP). Restriction enzymes digestion and sequencing were used to validate the recombinant plasmid (6).

\section{Cell culture}

MDA-MB-231 cell line, which is a human breast cancer cell line, was provided from Tehran Pasteur Institute (Tehran, Iran). Cells were preserved in a DMEM-Hi glucose medium (Gibco 31966-047), and 10\% fetal bovine serum was used as the growth supplement(FBS Gibco BI102-100) with penicillinstreptomycin (100 units $/ \mathrm{mL}$ ) at $37^{\circ} \mathrm{C}$ in a humidified incubator with $5 \% \mathrm{CO} 2$.

\section{Animal model}

Athymic female nude mice ( 6 weeks old and weighed 18-22g) were provided by Pasteur Amol Institute (Amol, Iran) Laboratory Animal Center. MDA-MB-231 cells were injected into 6-8 week old mice using Matrigel (50:50), which were kept in the Animal Pasteur Amol Institute. Throughout the study, the standard condition was maintained for the mice in the following way, the temperature of $25 \pm 2^{\circ} \mathrm{C}$, humidity within the range of $40 \%-60 \%$, and12L/12D light cycle in specific pathogen free housing. Initially, the nude mice skin was sanitized with $75 \%$ ethanol (9), $1 \times 10^{6}$ cells in $0.1 \mathrm{ml}$ of DMEM were then injected subcutaneously into the lower right hind flank of nude mice using a sterile syringe (10). After that, any sign of disease, including subcutaneous tumors or weight loss due to potential tumor growth in internal sites, were tracked in mice. Moreover, during the study, the mice were monitored for the growth of tumors, and tumor volume measurement was performed using calipers every three days. In this regard, tumor volume was obtained as $\pi \mid s^{2} / 6$, where I represents the long side and "s" demonstrates the short side (9). All nude mice were sacrificed by spinal cord injury.

\section{Plasmid treatment}

Based on previous studies we used 15 nude mice. When tumor diameters reached a size of about $8 \mathrm{~mm}$. (9), mice were assigned into three groups ( $n=5$ for each): 1 ) vector containing the genes group which 
received a subcutaneous injection of plasmid construct and jet polyethyleneimine (jetPEl; Polyplus Co., France); 2) control group which received subcutaneous injection with empty vector and jetPEI 3) reagent group which were injected with jetPEl, according to the protocol. The plasmids were administered three times a week for two weeks ( 6 total injections), and Caliper measurements of tumor size were performed. At $28 \mathrm{~d}$ following injection, the experiments were terminated, and all nude mice were sacrificed by spinal cord injury, and tumors were excised and weighed. It is worthy to note all animal experiments were performed following the 'Guide for the Care and Use of Laboratory Animals' published by the National Institutes of Health and were approved by the "Animal Care and Use Committee "of our university.

\section{Immunohistochemistry assessment of FOXO3a and P27kip1 expression}

In an attempt to determine the expression of FOXO3a and P27kip1, tumor samples were exposed to immunohistochemical examination. Tissue fixation was performed at $4^{\circ} \mathrm{C}$ using $4 \%$ paraformaldehyde before paraffin embedding. After that, they were cut into $5 \mu \mathrm{m}$ sections and transferred to silicon-coated slides, which were then stained with a monoclonal antibody against FOXO3a (Santa Cruz Biotechnology) at a dilution of 1:30 and use monoclonal antibody against P27kip1 (Santa Cruz Biotechnology) at a dilution of 1:100. The 3, 3'-diaminobenzidine tetrahydrochloride (DAB) was utilized for visualization, and Mayer's hematoxylin was used for counterstaining. Light microscopy was utilized for the evaluation of FOX03a and P27kip1 immunostained slides with a total magnification of 400x and a 10x10 square grid placed in the ocular. When tumor cells showed a distinct cytoplasmic and nuclear reaction, they were regarded as positive. The positive tumor cells were counted in 500 tumor cells in continuous high power. FOXO3a and P27kip 1 were determined by counting 500 tumor cells and were calculated as the percentage of positively labeled cells (8).

\section{Statistical analysis}

The data were analyzed in GraphPad Prism software (version 5.03, GraphPad, San Diego, CA, USA) using a 2-sided Student's paired t-test for single comparisons and one-way ANOVA with LSD posthoc test for multiple comparisons. All data were expressed as Mean \pm SEM. Moreover, Bonferroni's correction was used to adjust for multiple comparisons. A P value less than 0.05 was considered statistically significant.

\section{Results}

\section{Antitumor Activity of shFOXO3a and induced P27}

Based on the obtained data, the constructed plasmid resulted in tumor growth inhibition in vivo. The nude mouse tumor models were established by subcutaneous inoculation of $1 \times 10^{6}$ cells. Before the administration of plasmids or vehicle, the tumors were allowed to reach $8 \mathrm{~mm}$ in diameter. As depicted in Figure2, a significant reduction in tumor growth was observed in mice treated with construct plasmid, as compared to treatment with the negative control or the reagent (Figure 2). Besides, significant differences in tumor volumes were detected between the treated group (5/15) and controls group (10/15:empty 
vector and reagent), 14 days after the injection, $447 \pm 17.72 \mathrm{~mm} 3$, and $(662 \pm 36.1,608 \pm 19.3) \mathrm{mm} 3$, respectively $(P<0.05$, Figure 1$)$.

Nonetheless, no significant difference was reported between the empty vector control group and the reagent group. Accordingly, the treatment of construct plasmid halted tumor growth of cancer cells in nude mice. H\&E staining was used to prove the tumor was cancerous. It includes mitotic changes, severe eosinophilic cytoplasm, and increase the ratio of the nucleus to the cytoplasm (Figure 3). Percentage cancer cells of nude mouse tumor tissues that were staining with anti-P27 presented that it was $32.6 \%$ of construct compared with empty vector and reagent respectively $65.8 \%$ and $63.5 \%, p<0.05$ and with antiFOXO3 monoclonal antibody it was $22.1 \%$ of construct compared with empty vector and reagent $58.5 \%$ and $56.2 \%$ respectively (Figure 4,5$), p<0.05$.

\section{Discussion}

Triple-negative breast cancer (TNBC) is a challenging and aggressive type of breast cancer associated with a poorer prognosis, along with a higher risk of recurrence and metastasis (11). The present study aimed to examine the possibility of using a construct as a therapeutic agent against breast cancer. RNA interference (RNAi) is an evolutionarily conserved mechanism for specific gene silencing (12). Studies revealed that the transfection of the construct into mammalian cells could efficiently inhibit cancer cells. The tumor was generated in nude mouse models through subcutaneous inoculation of breast cancer cell lines. The findings of the experiment and immunohistochemical results were indicative of significant attenuation of tumor growth by construct was. Moreover, the results suggested that shRNA against the FOX03a gene and overexpression of P27 could significantly suppress the proliferation of breast cancer cell lines. Also, the results of a study conducted by Spratt et al. indicated that the decrease of P27 in a mouse model led to lung cancer (13). Storz et al. revealed that knockdown of FOXO3a resulted in decreased tumor size; moreover, they noted that any therapies involving the inactivation of FOXO3a might effectively block tumor expansion and metastasis(14). Besides, the obtained results of the present study data suggested that delivery of construct inhibits proliferation of breast cancer cell line leading to a decreased number of breast cancer and suppression of tumor cell growth in nude mice. Therefore, the current study suggested that silencing FOXO3a and simultaneous induction of P27 significantly contribute to the regulation of breast cancer cell line, growth antitumor activity against breast cancer.

In conclusion, the results were suggestive of the enormous impact of construct delivery on the ability to grow in vivo, suppression of breast cancer proliferation in vivo, and its effectiveness in breast cancer treatment. It is suggested that future studies focus on the efficiency of FOXO3a silencing and induced P27 as a novel biotherapy strategy for breast cancer patients.

\section{Conclusions}


In summary, the present study described that the construct we designed can suppress tumor growth in a nude mouse. Consequently gene delivery strategy, seems a practical therapeutic approach for breast cancer treatment.

\section{Abbreviations}

TNBC: Triple-negative breast cancer

ER: Estrogen Receptor

F0X03: Forkhead box 03

shRNA: short hairpin RNA

DAB: Diaminobenzidine tetrahydrochloride

RNAi: RNA interference

\section{Declarations}

Ethics approval and consent to participate: Iran. Golestan University of Medical Sciences. Research Ethics Committee (IR.goums.REC). 1394.150

It is worthy to note all animal experiments were performed following the 'Guide for the Care and Use of Laboratory Animals' published by the National Institutes of Health and were approved by the "Animal Care and Use Committee "of our university.

Consent for publication: Not applicable' for that section.

Availability of data and materials: Not applicable' for that section.

Competing interests: The authors declare that they have no competing interests.

Funding: This work was supported by the Golestan University of Medical Sciences (Grant number: 940714170) and Arya Tina Gene (ATG) Biopharmaceutical Company, Gorgan, Iran.

Role the funder(s): purchasing raw materials and do projects in laboratories there

Authors' contributions :

SM: Conducted the experiments, Data collection, Data analysis and interpretation, wrote the manuscript.

MG : Analyzed the results,Critical revision of the article, Final approval of the version to be published. 
MA: Analyzed the results of immunohistochemical and H\&E staining, Critical revision of the article, Final approval of the version to be published.

MSh: Analyzed the results, Design the study, Critical revision of the article , Final approval of the version to be published.

All Authors' Acknowledgment: that all authors have contributed significantly, all authors have read and approved the manuscript.

Acknowledgements:

We thank Golestan University of Medical Sciences and Arya Tina Gene (ATG) Biopharmaceutical Company, Gorgan, Iran. We also thank Pasteur Amol Institue for the parches and maintenance of mice.

\section{References}

1- See-Hyoung Park, Young Min Chung, Jessica Ma, Qin Yang, Jonathan S. Berek, Mickey C-T. Hu. Pharmacological activation of FOXO3 suppresses triple-negative breast cancer in vitro and in vivo. Oncotarget. 2016; 7: 42110-125. https://doi.org/10.18632/oncotarget.9881

2- Simon Taylor, Matthew Lam, Chathyan Pararasa, James EP Brown, Amtul R Carmichael and Helen R Griffiths. Evaluating the evidence for targeting FOXO3a in breast cancer: a systematic review. Cancer Cell International. 2015; 15: 1-9

3- Giuseppe Viglietto, Maria Letizia Motti, Paola Bruni, Rosa Marina Melillo, Amelia D’alessio, Daniela Califano. et al. Cytoplasmic relocalization and inhibition of the cyclin dependent kinase inhibitor p27Kip1 by PKB/Akt-mediated phosphorylation in breast cancer. NATURE MEDICINE. 2002; 8:1136-144. doi: 10.1038/nm762. Epub 2002 Sep 16.

4- Alkarain, R. Jordan, and J. Slingerland. p27 Deregulation in Breast Cancer: Prognostic

Significance and Implications for Therapy. Journal of Mammary Gland Biology and Neoplasia. 2004; 9: $67-80$

5- Peter Storz, Heike Do ppler, John A. Copland, Kaylene J. Simpson, and Alex Toker. FOXO3a Promotes Tumor Cell Invasion through the Induction of Matrix Metalloproteinases. MOLECULAR AND CELLULAR BIOLOGY, 2009; 29: 4906-17

6- Sabah Mayahi, Masood Golalipour, Ahad Yamchi, Gagan Deep Jhingan and Majid Shahbazi. New insights into the roles of the FOXO3 and P27Kip 1 genes in signaling pathways. UPSALA JOURNAL OF MEDICAL SCIENCES. 2019; 3:149-157. https://doi.org/10.1080/03009734.2019.1623351

7- Kun-Ming Rau, Chi-Ping Day, and Mien-Chie Hung. Breast Cancer Gene Therapy. Chapter 34: 705-40 
8- Nils Briinner, Birgitte Boysen, John Romer, and Mogens Spang Thomsen. The nude mouse as an in vivo model for human breast cancer invasion and metastasis. Breast Cancer Research and Treatment.1993;

24: $257-64$

9- Dong Liang, Min Dong, Lin-Jie Hu1, Ze-Hui Fang, Xia Xu1, En-Hui Shi, Yi-Ju Yang.

Hiwi Knockdown Inhibits the Growth of Lung Cancer in Nude Mice. Asian Pacific Journal of Cancer Prevention, 2013; 14: 1067-72

10- L. Bao, Y. Matsumura, D. Baban, Y. Sun, D. Tarin. Effects of inoculation site and Matrigel on growth and metastasis of human breast cancer cells. Br. J. Cancer.1994; 70: 228-232

11- Madeleine J. Oudin, Lucie Barbier, Claudia Schafer,Tatsiana Kosciuk, Miles A. Miller,

Sangyoon Han, et al. MENA Confers Resistance to Paclitaxel in Triple Negative Breast Cancer. Cancer Biology and Signal Transduction, 2016; 16: 143-155. DOI: 10.1158/1535-7163.MCT-16-0413

12- Connor Phalon, Donald D. Rao and John Nemunaitis. Potential use of RNA interference in cancer therapy. 2010; 12: 1-15

13- KS Kelly-Spratt, J Philipp-Staheli, KE Gurley, K Hoon-Kim, S Knoblaugh and CJ Kemp. Inhibition of PI$3 K$ restores nuclear p27Kip1 expression in a mouse model of Kras-driven lung cancer. 2009; 28: 3652-62 14- Peter Storz, Heike Do“ppler, John A. Copland, Kaylene J. Simpson, and Alex Toker. FOXO3a Promotes Tumor Cell Invasion through the Induction of Matrix Metalloproteinases. 2009; 29: 4906-17

\section{Figures}
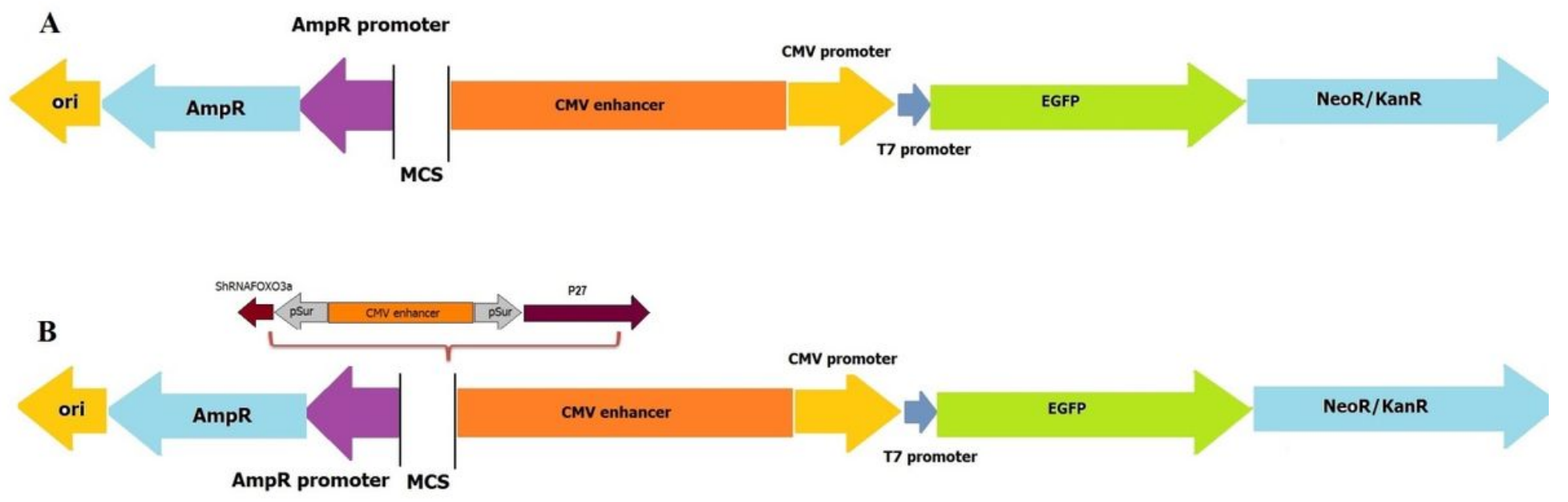
Figure 1

(A) Map of Empty vector; (B) Map of Construct vector


Figure 2

(A) Tumor growth curve of the three groups. (B) Weight of tumors from 3 groups of nude mice. Data represent Mean \pm SEM of five samples. $(P<0.05)$ 


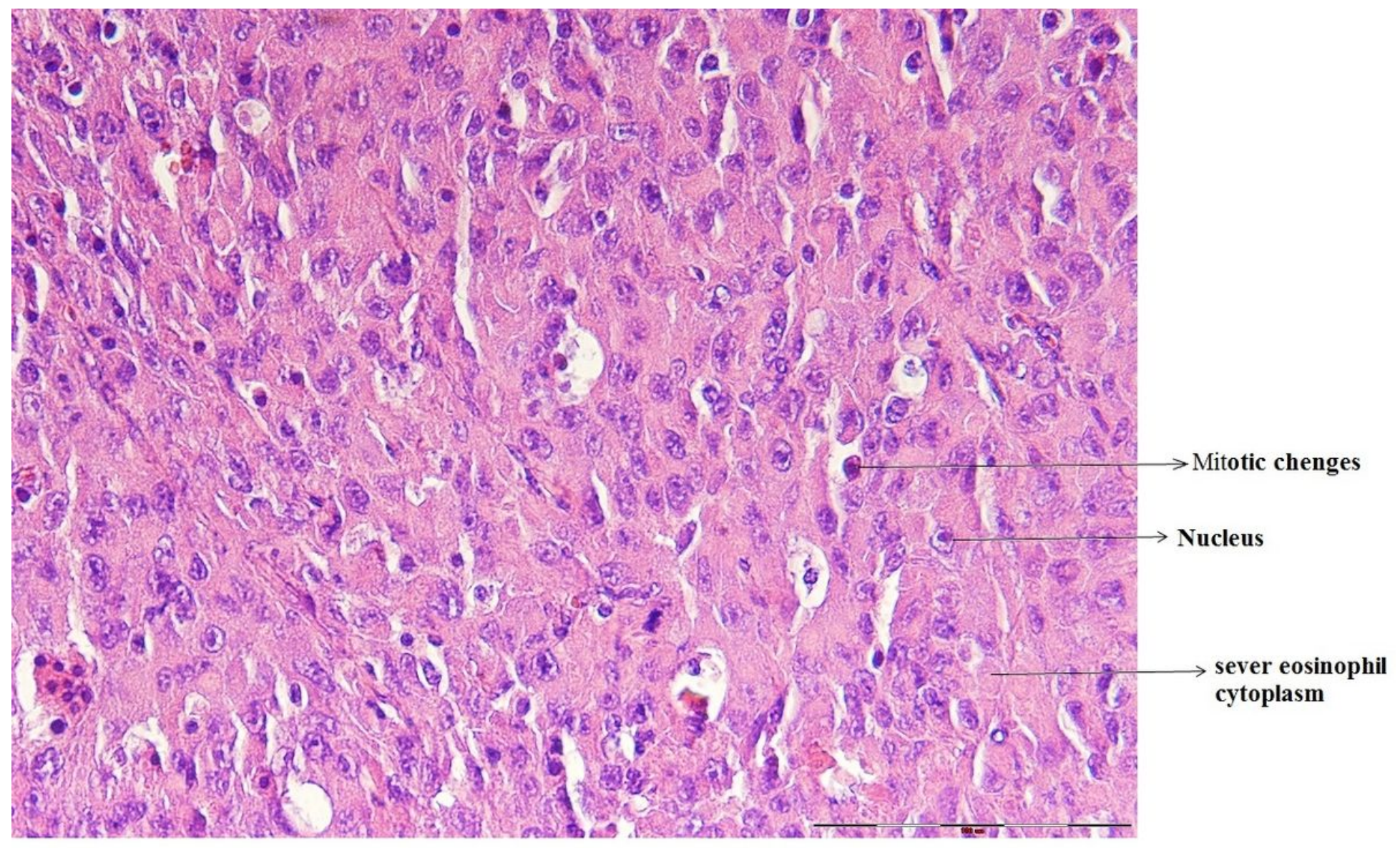

Figure 3

H\&E staining of tumor 
A

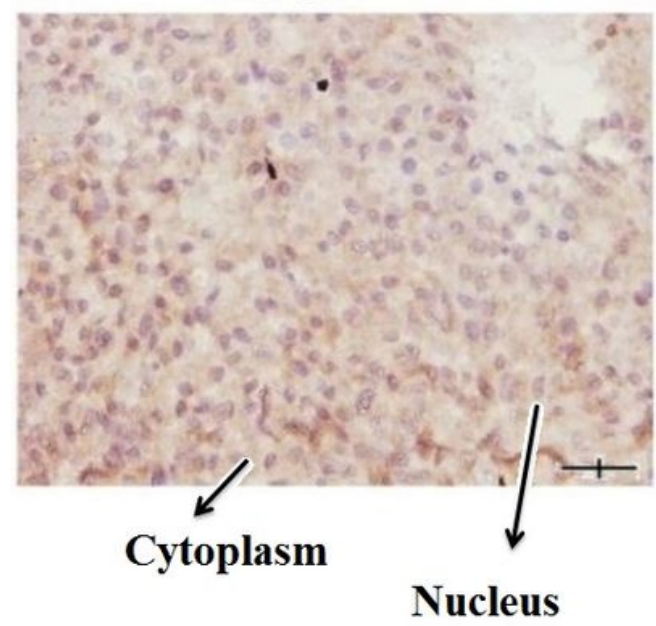

Empty Vector

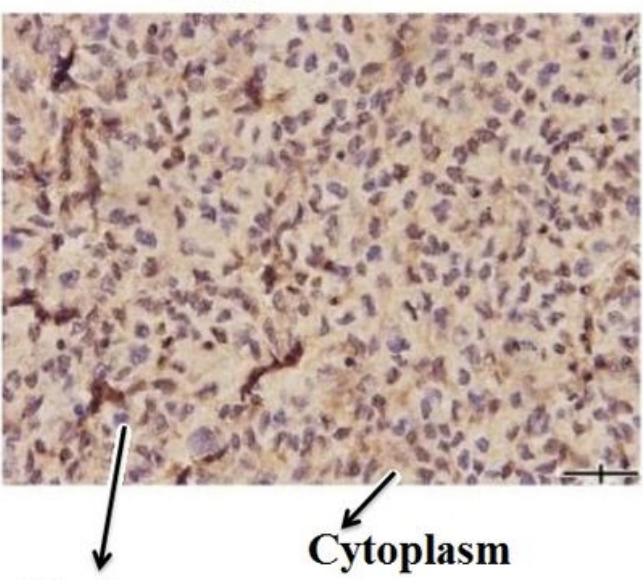

Vector containing gene

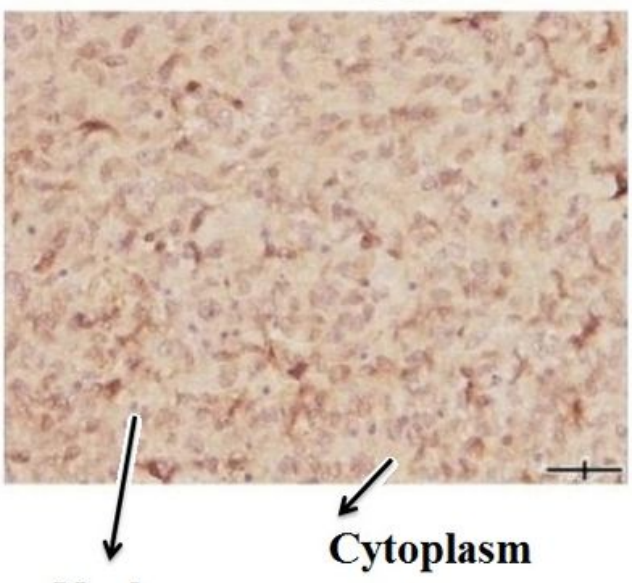

Nucleus

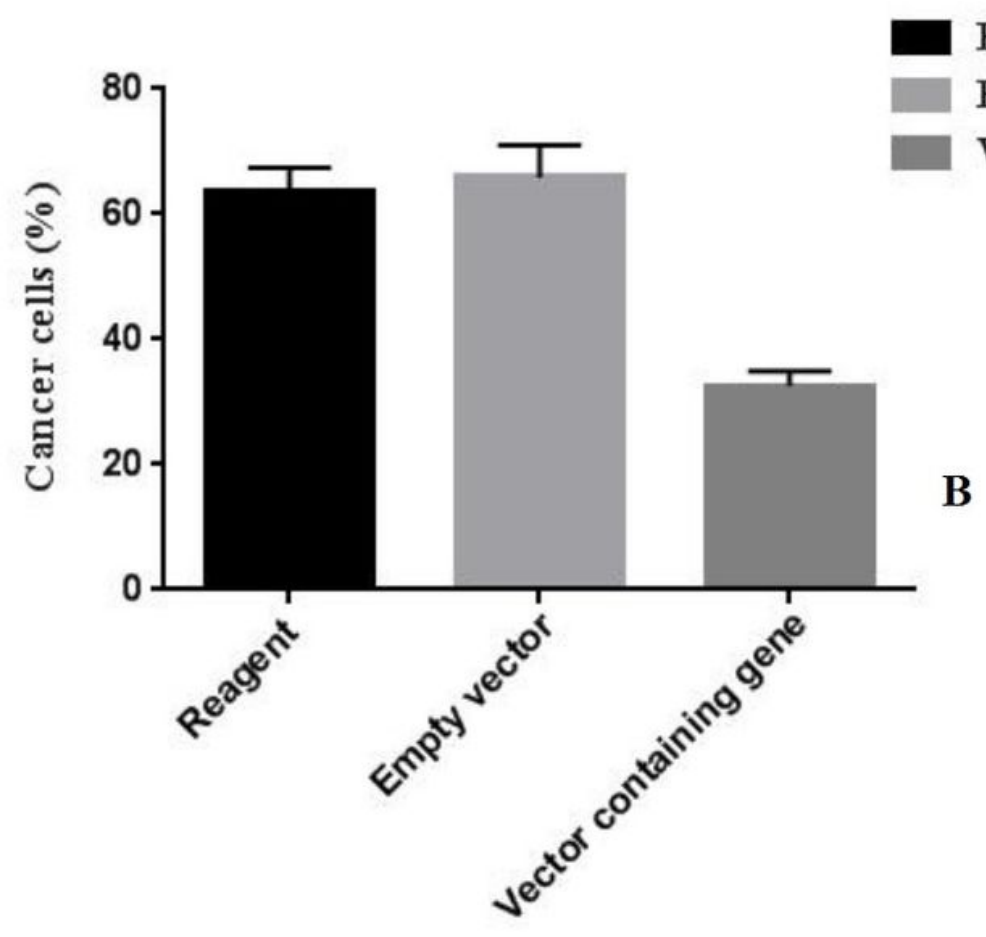

Figure 4

Immunohistochemical staining of P27 expression in tumors; (A) staining of nude mouse tumor tissues with the anti-P27 monoclonal antibody, revealing P27 expression in tumor tissues as a positive reaction in the cytoplasm (400X). The mice were treated with negative controls (empty vector and reagent) and construct, (B) Evaluation of immunostained slides under a light microscope (400X) using a 10×10 square grid (Positive cancer cell percentage was estimated by 500 tumor cells $100 \%$ ). Results are presented as mean $\pm S E M$. The controls and construct groups were compared at a significance level of $P<0.05$. 
$\mathbf{A}$

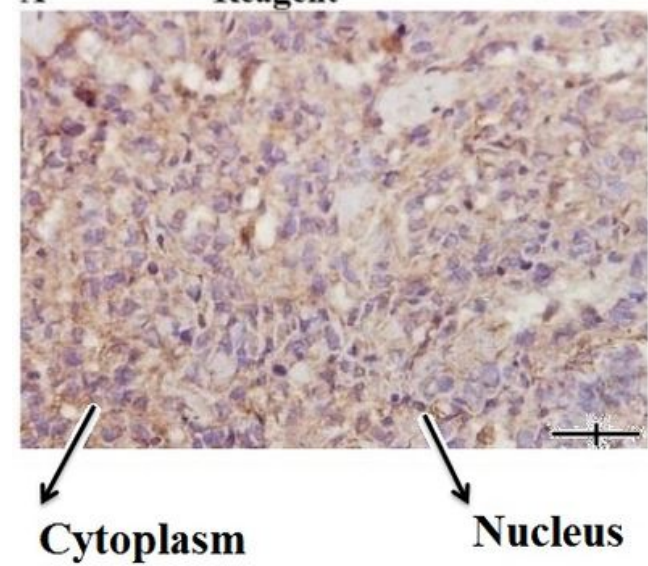

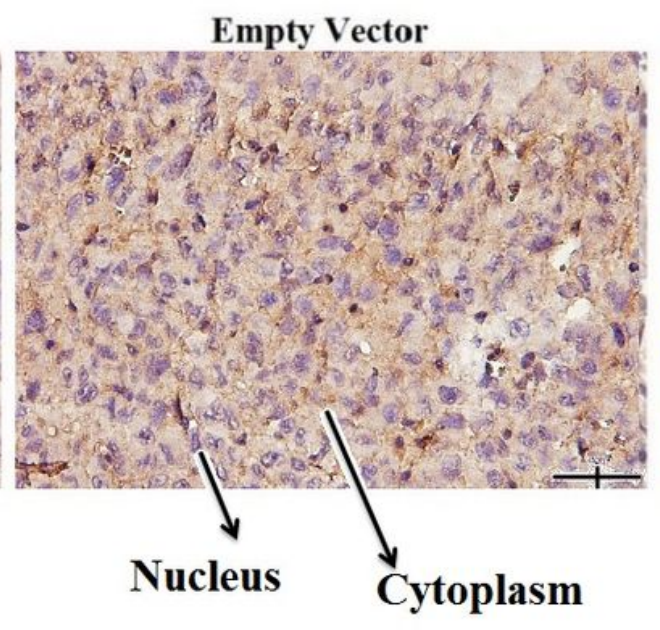

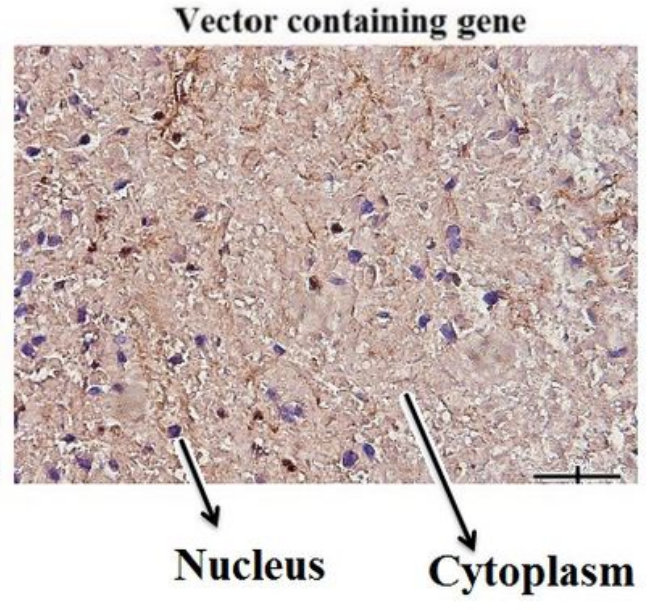

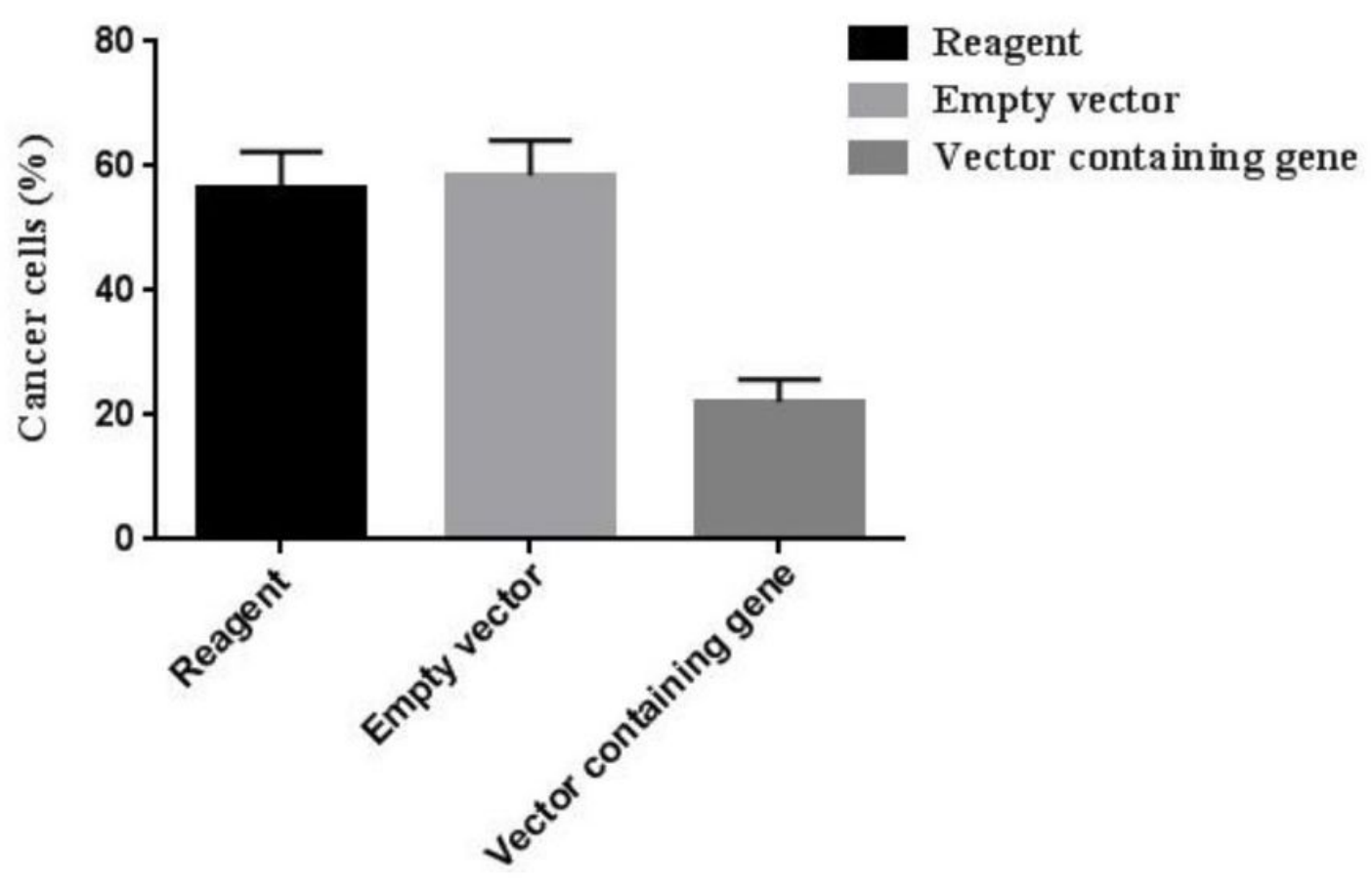

Figure 5

Immunohistochemical staining of FOXO3 expression in tumors; (A)staining of nude mouse tumor tissues with an anti-FOXO3 monoclonal antibody, exhibiting FOXO3 expression in tumor tissues as a positive reaction in the cytoplasm (400X). The mice were treated with negative controls (empty vector and reagent) and construct, (B) evaluation of immunostained slides under a light microscope (400X) using a $10 \star 10$ square grid (The positive cancer cell percentage was estimated by 500 tumor cells $100 \%$ ). The results are presented as mean $\pm S E M$. The control and construct groups were compared at a significance level of $\mathrm{P}<0.05$. 


\section{Supplementary Files}

This is a list of supplementary files associated with this preprint. Click to download.

- supplement9.pdf 